\title{
The Many Faces of Acute Idiopathic Blind Spot Enlargement
}

\author{
Amiee Ho, OD \\ Assistant Professor, \\ Pacific University \\ College of Optometry \\ Denise Goodwin, OD, FAAO \\ Professor, \\ Pacific University \\ College of Optometry
}

\begin{abstract}
This is a literature review and retrospective case series of acute idiopathic blind spot enlargement in two Caucasian females and one Hispanic male. Hallmark findings include unilateral photopsia, an enlarged blind spot, loss of the ellipsoid and interdigitation zones on optical coherence tomography (OCT), and an abnormal multifocal electroretinography. This condition is an outer retinal disease that shares common traits with the acute zonal occult outer retinopathy (AZOOR) complex, which can confound the diagnosis. Autofluorescent imaging may aid in differentiating the conditions. Dissemination of more case reports detailing different presentations of this rare outer retinal disorder should help advance the clinical understanding of these conditions.
\end{abstract}

\section{KEYWORDS:}

Acute idiopathic blind spot enlargement; acute zonal occult outer retinopathy; optic nerve; outer retinal disease; photopsia; visual field

\section{INTRODUCTION}

Acute idiopathic blind spot enlargement (AIBSE), first described in $1988,{ }^{1}$ is a rare condition that affects the peripapillary retina. It results in an acuteonset scotoma contiguous with the physiological blind spot despite a lack of commensurate optic nerve swelling. The scotoma varies in size but generally is an absolute defect with steep margins that does not progress over time. Fundus examination is often normal or only slightly abnormal, and therefore the condition is often diagnosed incorrectly. ${ }^{2-4}$ It is commonly misdiagnosed as optic neuritis due to the demographics and vision loss despite relatively normal ocular health or as a migraine aura due to photopsias.

The etiology of AIBSE is unknown. Multiple mechanisms have been proposed for outer retinal diseases, including an infectious or autoimmune etiology. ${ }^{5}$ Hormonal or genetic factors may play a role. ${ }^{4}$ Patients are typically female between the ages of 19 and 53 years; ${ }^{4}$ however, the condition has been reported in males. ${ }^{1,3,6}$ Ethnicity is often not reported in the available case studies, ${ }^{4,7}$ but AIBSE has been reported in Caucasians ${ }^{8}$ and Asians. ${ }^{3,6}$

In the largest series to date, Volpe et al. ${ }^{4}$ described 27 patients with AIBSE collected over an eight-year period. The most common symptom in patients with AIBSE was decreased vision (92\%), categorized as blur, missing portions of vision, spots in the vision, or the feeling of looking through a film. ${ }^{4}$ Other symptoms include positive visual phenomenon (85\%), such as photopsia, swirling, movement within the scotoma, colored lights, or a phenomenon similar to the afterimage seen after looking at a flash bulb. Visual acuity is normal in $60 \%$ of patients and, if abnormal, is between $20 / 25$ and 20/50 in nearly all patients. ${ }^{4}$ Other clinical findings include mild optic nerve swelling (44\%), dyschromatopsia (33\%), relative afferent pupillary defect (30\%), peripapillary pigment changes (22\%), white retinal dots (18\%), and peripapillary subretinal greyish discoloration (15\%). ${ }^{4}$ Over time, chorioretinal scarring that corresponds to the visual field defect is likely to develop. ${ }^{9}$

Optical coherence tomography (OCT) will show loss of the ellipsoid zone and interdigitation line in the involved peripapillary area. Multifocal electroretinography is essential in making the diagnosis of AIBSE. A full-field electro- 
retinogram will often be normal ${ }^{4}$ because it takes an average of the overall retinal activity, and the healthy retina will mask the abnormal readings. A focal or multifocal electroretinogram will demonstrate localized abnormalities that correlate with the visual field loss. The abnormalities on multifocal electroretinography will persist after other signs have resolved, and there may be bilateral abnormalities despite an otherwise unilateral presentation. ${ }^{9}$

Differential diagnoses that result in an enlarged blind spot include optic nerve head swelling (for example papilledema) causing anterior displacement of the peripapillary retina. In these cases, the size of the blind spot corresponds to a visible abnormality. Demyelinating optic neuritis, which is most common in young females, is an atypical cause of an enlarged blind spot. Ocular health is normal in two-thirds of these cases due to retrobulbar involvement. There is generally pain with eye movements, a significant relative afferent pupillary defect, and color vision deficits. OCT can be helpful in differentiating demyelinating optic neuritis from AIBSE, as optic neuritis involves the inner retina (retinal ganglion cell and nerve fiber layer) and should not cause disruption of the outer retinal layers. With demyelinating optic neuritis, the results of multifocal electroretinography will be normal, since the test measures outer retinal function. Other differential diagnoses include autoimmune retinopathies, such as cancer-associated retinopathy or melanoma-associated retinopathy, in which the fundus appears normal in early cases. These conditions will have a slower onset compared with AIBSE and progress without intervention. ${ }^{5}$ Additionally, high myopia can cause a slightly enlarged blind spot along with visible peripapillary abnormalities and symptoms of photopsia.

An enlarged blind spot due to outer retinal disease may be isolated, as in AIBSE, or associated with other conditions, including multiple evanescent white dot syndrome (MEWDS), acute macular neuroretinopathy, multifocal choroiditis, punctate inner choroidopathy, acute annular outer retinopathy, and acute zonal occult outer retinopathy (AZOOR). Overlap between these conditions, all of which affect the outer retina and occur primarily in young or middle-aged women, has caused many to question whether the conditions are all variations of a single disease known as the AZOOR complex..$^{3-5}$ Improved imaging devices and attention to minor differences may help distinguish the conditions.

There is no proven treatment for AIBSE. Photopsia should decrease over 3 to 4 months. The visual field loss may regress slightly, but generally does not completely resolve, although there are some reports to the contrary.,9,10 The first report describing AIBSE reported resolution of symptoms within 2 to 3 months and a normal blind spot in 3 of the 7 patients. ${ }^{1}$ It has been argued that some of these patients may have represented late cases of MEWDS in which the fundus lesions had resolved. ${ }^{8}$ Volpe et al. ${ }^{4}$ followed-up 10 of the 27 patients in their case series; all had a decrease in photopsia, but there was no improvement in the blind spot in any of the 10 patients. Multifocal electroretinography defects may persist even if the visual field defect resolves and can be helpful in detecting subclinical retinal dysfunction. ${ }^{8}$ Recurrence is possible, albeit rare.

Here we present three varying presentations of AIBSE to add to the existing case reports. The first case is a classic presentation of the condition. The second case, to the best of our knowledge, is the first reported case in a Hispanic male. The third case demonstrates the potential intermittent nature of the condition and has not yet been reported. These examples of this rare condition may help further define and classify the diagnosis of AIBSE.

\section{CASE REPORTS}

Case 1: A 28-year-old Caucasian female was referred to rule out demyelinating optic neuritis. She had sudden-onset, constant blurriness, and flashes of light in the temporal side of the vision in the left eye for two weeks. She had not experienced any recent fever or illness, she was not taking any medications, and she reported no pain with eye movements. Visual acuities were 20/20 right eye and 20/20-1 left eye. There was a mild relative afferent pupillary defect in the left eye. Pseudoisochromatic plates showed a mild red-green defect and $20 \%$ red desaturation in the left eye. The left fundus had mildly indistinct disc margins with a scleral and choroidal crescent (Fig. 1A). An optic nerve head OCT showed slightly increased peripapillary retinal nerve fiber layer thickness inferiorly in the left eye, and a macular OCT showed loss of the outer nuclear layer, external limiting membrane, ellipsoid zone, and interdigitation zone extending one-third of the distance between the temporal margin of the optic disc toward the fovea (Fig. 1B). Visual field analysis (Fig. 1C) demonstrated a deep temporal scotoma surrounding the blind spot in the left eye. Magnetic resonance imaging (MRI) with contrast did not show any evidence of optic neuritis or demyelination. Multifocal electroretinography (Fig. 1D) showed significantly reduced amplitude and implicit time in the area corresponding to the visual field loss. Eight weeks later, the patient no longer experienced photopsia, but the visual field defect and outer retinal loss on OCT were stable. The left relative afferent pupillary defect remained. 
Figure 1: A 28-year-old Caucasian female with AIBSE in the left eye has mildly indistinct disc margins (A), loss of outer retinal layers adjacent to the optic nerve head on macular OCT (B), an enlarged blind spot $(C)$, and significantly reduced amplitude and implicit time in the involved area with $\mathrm{MfERG}(D)$. All investigations of the uninvolved right eye were unremarkable.
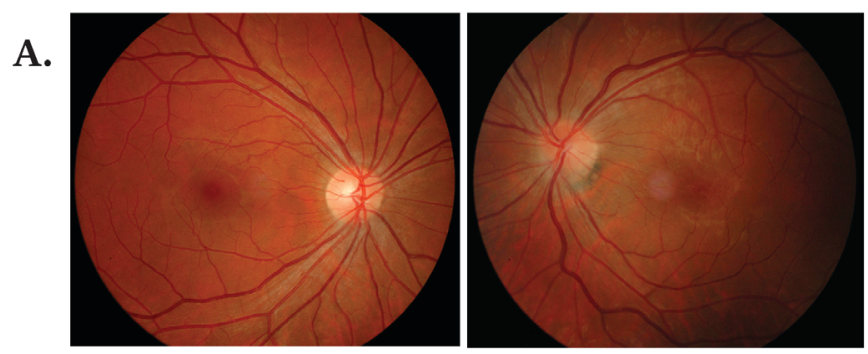

B.
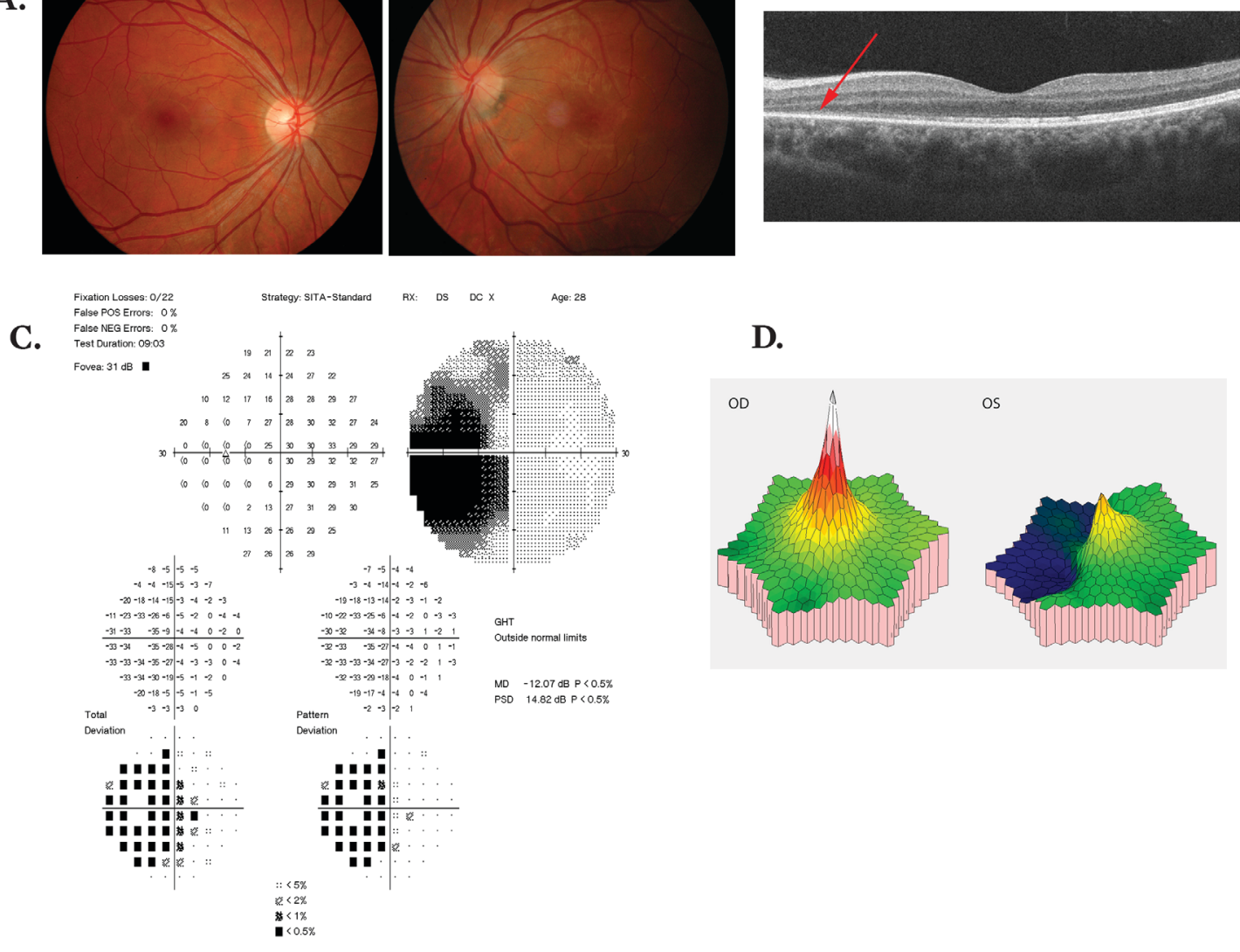

D.

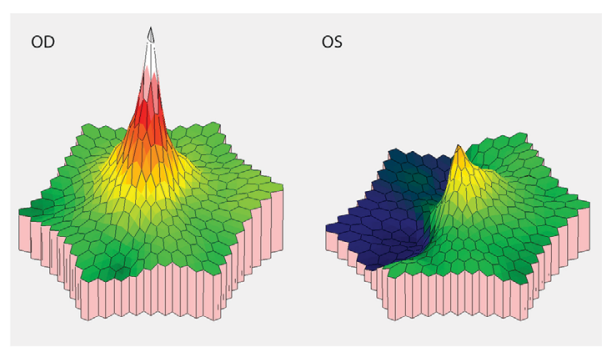

Case 2: A 24-year-old Hispanic male had noted a constant circular black spot in the upper left corner of his vision nine days earlier. When he closed his eyes, the spot appeared as a greenish white light. He had not experienced a recent illness or flu-like symptoms. Ocular and medical history were unremarkable. He was taking no medications. Best correct visual acuities were 20/15 right eye and 20/15 left eye. There was no relative afferent pupillary defect, and color vision was normal. Posterior segment examination revealed a non-tilted horizontally elongated disc with an inferior scleral crescent in both eyes (Fig. 2A). Macular OCT showed loss of the external limiting membrane, ellipsoid zone, and interdigitation zone temporal to the optic disc (Fig. 2B) in the left eye. Visual field analysis (Fig. 2C) demonstrated a deep temporal scotoma surrounding the blind spot in the left eye. Multifocal electroretinography (Fig. 2D) showed significantly reduced amplitude and mildly reduced implicit time in the area corresponding to the visual field loss. Six weeks later, he noticed the spot less if both eyes were open. The visual field and multifocal electroretinography were similar to the previous readings. Two years after the initial event, the patient reported that the spot was still present, but he did not notice it unless he closed the right eye. Although some differences could be attributed to using different testing strategies, a small scotoma contiguous with the blind spot in the left eye remained (Fig. 2E). The left eye showed significant hypoautofluorescence in the peripapillary area without any significant hyperautofluorescence (Fig. 2F). The area of decreased amplitude on multifocal electroretinography was smaller (Fig. 2G). No evident change was present on OCT. 
Figure 2: A 24-year-old Hispanic male with AIBSE in the left eye has horizontally elongated optic nerves with an inferior scleral crescent in both eyes (A), loss of outer retinal layers on macular OCT (B), an enlarged blind spot $(C)$, and significantly reduced amplitude and implicit time with $\mathrm{MfERG}(D)$ in the left eye only. Two years later, a persistent small scotoma remains in the left eye (E). Fundus autofluorescence $(F)$ shows hypoautofluorescence in the area of the scleral crescent in both eyes with a slight hyperautofluorescent crescent nasally in the right eye, and a smaller area of decreased amplitude remains in the left eye on $m f E R G(G)$.

A.

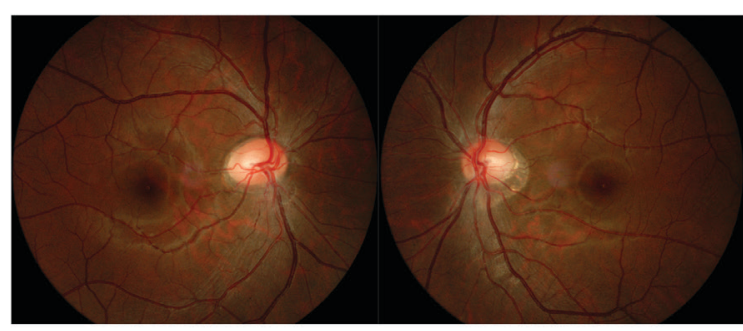

B.

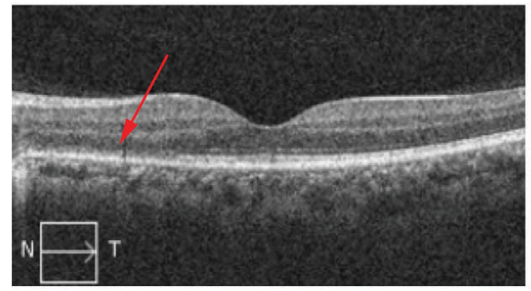

C.

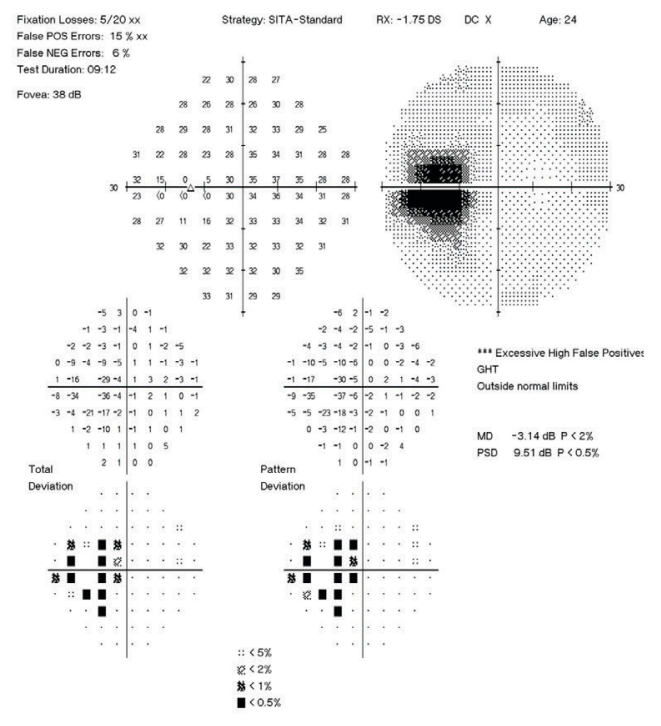

E.

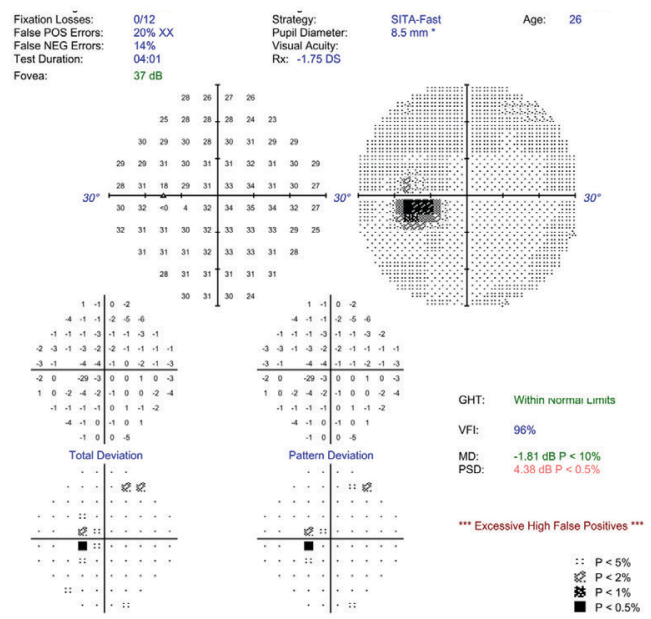

D.

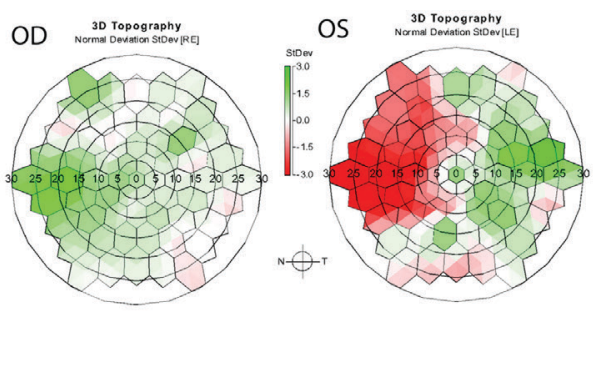

F.
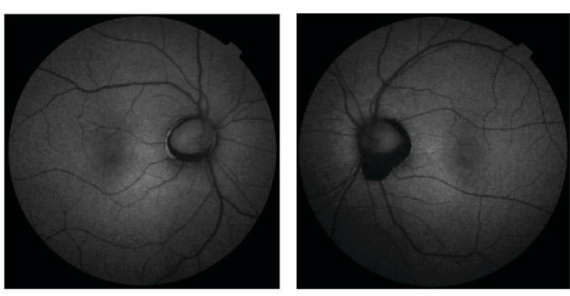

G.

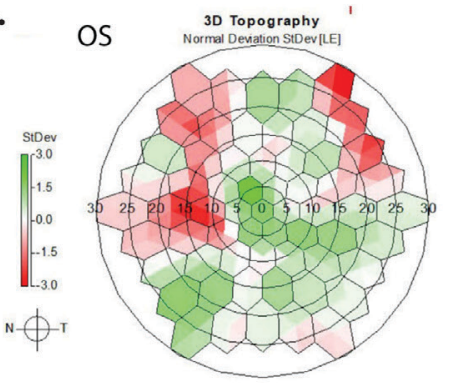


Case 3: A 24-year-old Caucasian female noticed a shimmering area in the temporal field of the right eye on and off for three years. The shimmering lasted about 15 seconds and was followed by a blind spot in the same area that would last a couple of hours. Medical health was unremarkable, and she was taking levonorgestrel/ethinyl estradiol. Best corrected visual acuity was 20/15 in each eye. There was no relative afferent pupillary defect, and color vision was normal. Before and after an episode, there was a 360-degree halo surrounding the right optic nerve (Fig. 3A). During an episode, the peripapillary halo became larger (Fig. 3B) and the blind spot was enlarged slightly in the right eye (Fig. 3C). Before and after the episode, the visual fields were unremarkable. Between episodes, a 5-line raster showed a small area of disruption of the ellipsoid and interdigitation zones temporal to the optic nerve in the right eye, whereas the corresponding area in the left eye was unremarkable (Fig. 3D). Multifocal electroretinography performed between episodes showed normal amplitude and implicit time; however, responses from the nasal retina of the right eye were reduced and slightly delayed relative to the remainder of the responses from that eye (Fig. 4E).

Figure 3: A 24-year-old Caucasian female with intermittent AIBSE in the right eye. Fundus photos before (A) and during (B) an episode show increased grey halo surrounding the optic nerve head during the episode. There is an enlarged blind spot in the right eye during an episode (C). There is a small area of disruption of the outer retinal layers just temporal to the optic nerve in the right eye $(D)$ and relatively reduced and slightly delayed responses in nasal retina of the right eye with $m f E R G(E)$.

A.

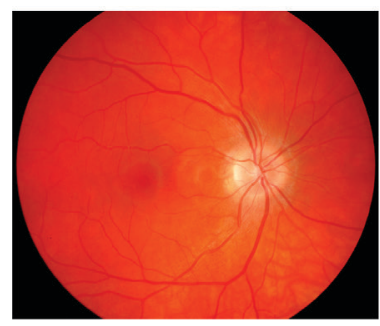

B.

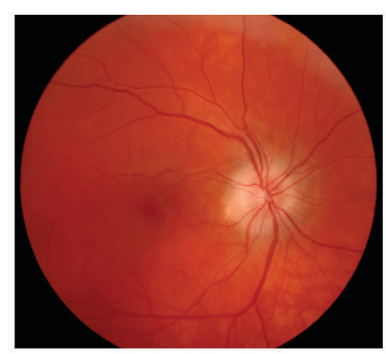

C.

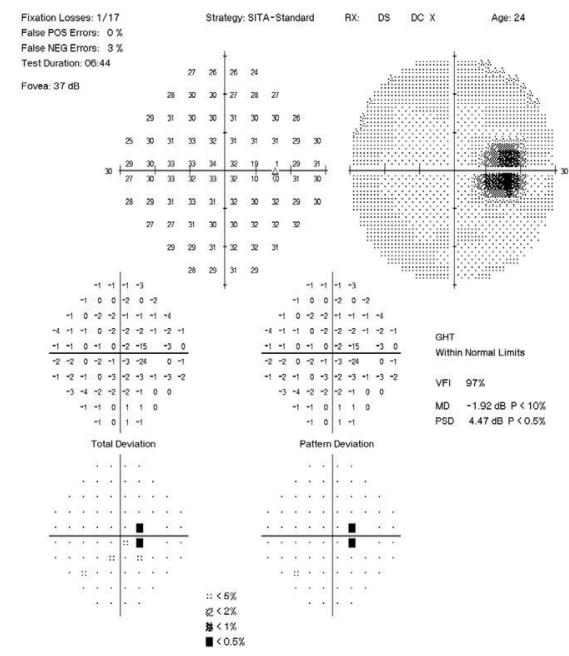

D.
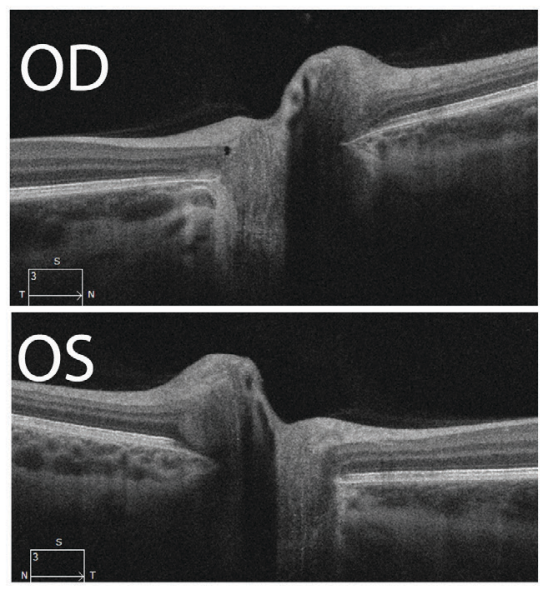

E.
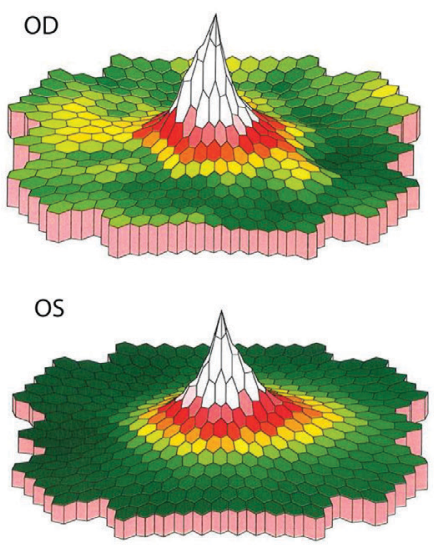
Figure 4: Multiple scattered white-dot lesions in the right eye (A) associated with MEWDS. Following resolution of the white dots, there is macular pigment granularity in the right eye $(B)$. Macular OCT $(C)$ shows involvement of the ellipsoid zone and interdigitation zone at the fovea of the right eye. Multifocal ERG (D) shows an area of reduced amplitude extending nasally from the fovea in the right eye.

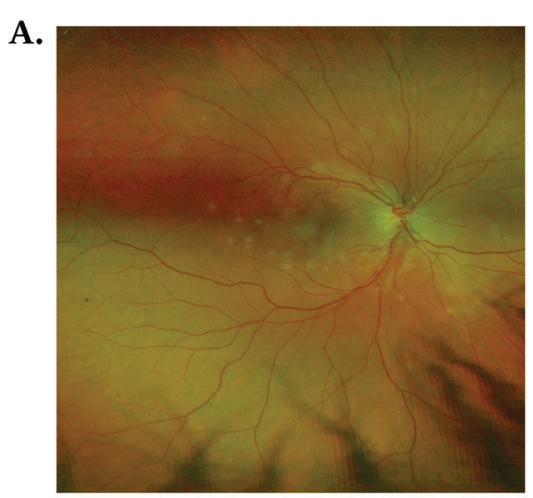

C.

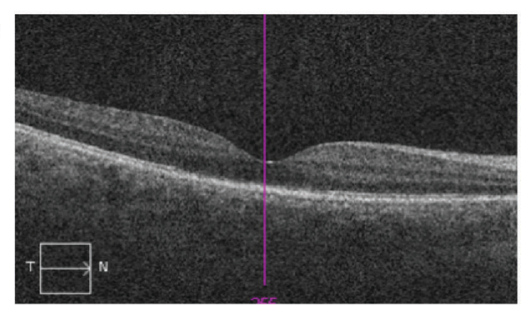

B.

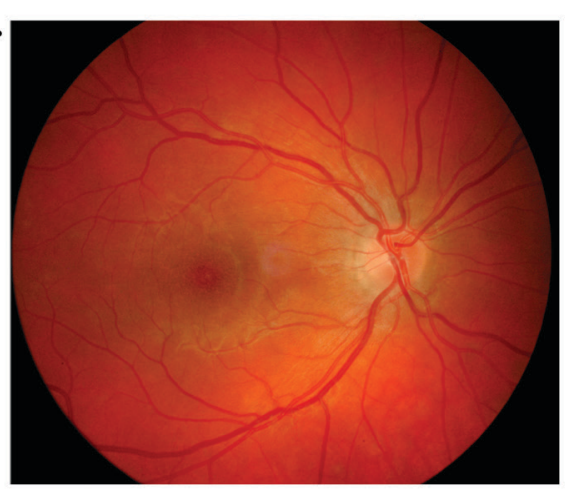

D.

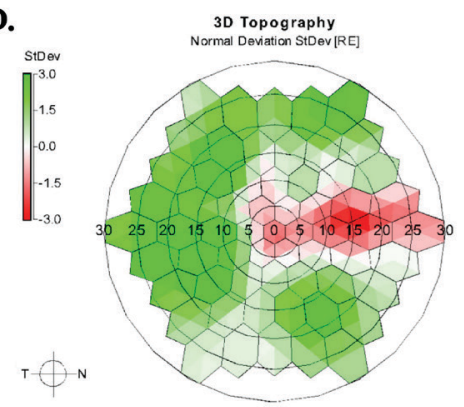

\section{DISCUSSION}

Acute idiopathic blind spot enlargement (AIBSE) is a rare condition. Here we add three cases of AIBSE to the existing literature. The first case is representative of a classic presentation of AIBSE, although it again verifies that the condition continues to be misdiagnosed, as it was initially diagnosed as demyelinating optic neuritis. The second and third cases had no diagnosis at the time of referral. The second case is unusual in that it occurred in a Hispanic male; most reported cases of AIBSE occur in young Caucasian females. To our knowledge, this is the first reported case of AIBSE in a Hispanic male patient. Finally, with the third case, we add an unusual presentation of intermittent AIBSE.

As seen in all three of these cases of AIBSE, the optic disc often has an abnormal appearance, such as congested disc or mild disc edema. ${ }^{9}$ Two of the three patients (Cases 1 and 3) had mild disc edema and a greyish peripapillary halo. Interestingly, the greyish area significantly enlarged during the intermittent events in Case 3 . Two of the cases involved a horizontally elongated nerve with significant peripapillary atrophy. In all three cases, peripapillary changes in the involved eye were greater than those in the non-involved eye.

Consistent with outer retinopathy, our presented cases showed abnormal multifocal electroretinography corresponding to the visual field loss. All cases showed both reduced amplitude and implicit time. Interestingly, in Case 3, which exhibited intermittent AIBSE, multifocal electroretinography showed abnormalities between episodes despite the patient having no symptoms or visual field loss. In addition to multifocal electroretinography defects, the other critical diagnostic feature of AIBSE, outer retinal abnormalities, including loss of the ellipsoid and interdigitation zones noted on OCT, was present in all patients.

There continues to be debate over whether AIBSE is a distinct condition or simply a subset of the AZOOR complex. AIBSE, MEWDS, and AZOOR share common features. The conditions are more common in females and present with photopsias. The visual field loss in AZOOR may or may not be contiguous with the blind spot. With AZOOR, there is generally progression of the visual field loss and retinal anomalies over weeks or months, whereas the visual field loss with AIBSE typically remains stable. The retinal defects are bilateral in 39\% of AZOOR cases, ${ }^{5}$ while AIBSE is generally unilateral.,6 Of our presented cases, all had non-progressive, unilateral scotoma, which is more consistent with AIBSE than with AZOOR.

The widespread availability of autofluorescence is relatively new, and therefore few cases demonstrate the defects produced by AIBSE. Areas of hyperautofluorescence indicate distress of the RPE while areas of hypoautofluorescence indi- 
cate death of RPE. Therefore, this technology may be helpful in differentiating AIBSE from AZOOR. With AZOOR, there will be a patchy hyperautofluorescent signal in the involved area that progresses over time. ${ }^{11}$ On fundus examination, there is a demarcating line around the involved area, typically orange in color, that is better seen on autofluorescent imaging. ${ }^{11}$ This hyperautofluorescent line which demarcates the normal and involved retina is a defining feature of AZOOR. ${ }^{11}$ In addition to frequent zonal progression, Mrejen and colleagues ${ }^{11}$ proposed the following fundus autofluorescence criteria to diagnose AZOOR: a narrow hyperautofluorescent demarcation line between the involved and uninvolved retina and a trizonal pattern of degeneration. The trizonal pattern can be seen on autofluorescence as an area of normal autofluorescence outside the involved region (zone 1), an area of hyperautofluorescence (zone 2), and an area of hypoautofluorescence (zone 3). In Case 2, in which autofluorescent imaging was performed, no hyperautofluorescent line demarcating the abnormal tissue or trizonal pattern was present. There was significantly more peripapillary hypoautofluorescence in the involved eye compared to the normal eye, corresponding to the area of peripapillary atrophy. The signal was brighter surrounding the optic nerve, but this appears to be equal in both eyes. In AZOOR patients, the trizonal pattern can also be seen on OCT as a normal area outside the involved tissue (zone 1), a zone of subretinal drusenoid deposits (zone 2), and a zone of loss of photoreceptors (zone 3). This pattern was not present in any of the AIBSE patients in this report. The lack of the trizonal pattern with either autofluorescence or OCT may lend credence to AIBSE being a separate condition.

AIBSE has features similar to MEWDS, and some argue that the characteristic white dots in the latter are not visible or have resolved in AIBSE. MEWDS is generally preceded by a viral-type illness and involves white lesions at the level of the photoreceptors that are visible for a few weeks (Fig. 4A). Vision normally recovers fully, but after resolution, the patient develops permanent macular pigment granularity that is considered pathognomonic of MEWDS (Fig. 4B). Despite seeing these patients in the acute stage, none had retinal white dots and all denied viral-type symptoms. In addition, macular granularity was not present in any of our cases.

It has been proposed that if the scotoma surrounding the blind spot regresses, this is suggestive of a diagnosis of MEWDS, whereas peripapillary atrophy along with scotoma persistence leads to a diagnosis of multifocal chorioretinitis, and progression of the scotoma is indicative of AZOOR. ${ }^{10}$ Unfortunately, this classification does not take into account other characteristic features. Multifocal chorioretinitis is associated with anterior uveitis, as well as the presence of multiple yellow-gray choroidal lesions that become punched out scars over time. Despite no resolution or progression, none of the three cases had retinal lesions consistent with these features of multifocal chorioretinitis.

\section{CONCLUSION}

Although AIBSE is poorly understood, it is important that eye care practitioners be familiar with its characteristics. Additional examples of this rare condition may help to define and classify the diagnosis. Our cases are not consistent with other outer retinopathies and may indicate that AIBSE is a separate condition from AZOOR or MEWDS. A nonprogressive unilateral enlarged blind spot with corresponding findings on multifocal electroretinography without other indications of other outer retinal disorders is consistent with a diagnosis of AIBSE. Generally, the patient will have acute symptoms of vision loss and photopsia within the temporal field. The symptoms generally improve over time, but the visual field and multifocal electroretinography abnormalities remain. •

CORRESPONDING AUTHOR:

Amiee Ho, O.D., amieeho@pacificu.edu

DISCLOSURE OF INTEREST:

Financial Support: None. The authors report no conflict of interest

REFERENCES

1. Fletcher WA, Imes RK, Goodman D, et al. Acute idiopathic blind spot enlargement. A big blind spot syndrome without optic disc edema. Arch Ophthalmol 1988;106:44-9.

2. Jiang L-B, Shen C-Y, Chen F, et al. Clinical features of retinal diseases masquerading as retrobulbar optic neuritis. Chin Med J 2013;126:3301-6.

3. Sugahara M, Shinoda K, Matsumoto SC, et al. Outer retinal microstructure in a case of acute idiopathic blind spot enlargement syndrome. Case Rep Ophthalmol 2011;2:116-22.

4. Volpe NJ, Rizzo JF, Lessell S. Acute idiopathic blind spot enlargement syndrome: a review of 27 new cases. Arch Ophthalmol 2001;119:59-63.

5. Monson DM, Smith JR. Acute zonal occult outer retinopathy. Surv Ophthalmol 2011;56:23-35.

6. Liu X, Chen B, Zhang M, et al. Clinical features and differential diagnosis of acute idiopathic blind spot enlargement syndrome. Eye Sci 2014;29:143-50.
7. Cooper ML, Lesser RL. Prolonged course of bilateral acute idiopathic blind spot enlargement. J Clin Neuroophthalmol 1992;12:173-7.

8. Kondo N, Kondo M, Miyake Y. Acute idiopathic blind spot enlargement syndrome: prolonged retinal dysfunction revealed by multifocal electroretinogram technique. Am J Ophthalmol 2001;132:126-8.

9. Watzke RC, Shults WT. Clinical features and natural history of the acute idiopathic enlarged blind spot syndrome. Ophthalmology 2002;109:1326-35.

10. Trese MGJ, Cohen SR, Besirli CG. Recovery of outer retina in acute idiopathic blind spot enlargement (AIBSE). Am J Ophthalmol Case Rep 2016;1:13-5.

11. Mrejen S, Khan S, Gallego-Pinazo R, et al. Acute zonal occult outer retinopathy: a classification based on multimodal imaging. JAMA Ophthalmol 2014;132:1089-98. 